\title{
Model-based Leakage Localization in Drinking Water Distribution Networks using Structured Residuals
}

\author{
Albert Rosich ${ }^{1}$ and Vicenç Puig ${ }^{2}$
}

\begin{abstract}
In this paper, a new model based approach to leakage localization in drinking water networks is proposed based on generating a set of structured residuals. The residual evaluation is based on a numerical method based on an enhanced Newton-Raphson algorithm. The proposed method is suitable for water network systems because the non-linearities of the model make impossible to derive analytical residuals. Furthermore, the computed residuals are designed so that leaks are decoupled, which improves the localization of leaks with respect to other existing methods. Finally, the Hanoi water network benchmark is used to illustrate the results of the proposed approach.
\end{abstract}

\section{INTRODUCTION}

Water loss in distribution networks, caused by accidentally pipe bursts or intentionally breakdowns, is an issue of great concern for water utilities, strongly linked with operational costs and water resources savings. Continuous improvements in water loss management are being applied and new technologies are developed to achieve higher levels of efficiency. Usually, a leakage detection method in a DMA (District Metered Area) starts by analyzing input flow data, such as minimum night flows and consumer metering data [9], [3]. After the water distribution district is identified to have a leakage, various techniques are used to locate it for pipe replacement or repair. Methods for locating leaks range from ground-penetrating radar to acoustic listening devices or physical inspection [5]. Some of these techniques require isolating and shutting down part of the system. The whole process could take weeks or months with a significant volume of water wasted. Techniques based on locating leaks from pressure monitoring devices allow a more effective and less costly search in situ.

The methodology of leakage localisation proposed in this paper is mainly based on standard theory of model-based diagnosis described for example in [7] that has already been applied to water networks to detect faults in flow meters [15] or in open channel with dynamic models [11]. The principle of model-based fault diagnosis is to check the consistency of observed behaviour with the a priori system information

*This work was supported by the Fonds National de la Recherche, Luxembourg, under the project C11/IS/1206050 (SeSaNet), by CICYT SHERECS DPI-2011-26243 and CICYT WATMAN DPI-2009-13744 of the Spanish Ministry of Education and by i-Sense grant FP7-ICT-20096-270428 of the European Commission.

${ }^{1} \mathrm{~A}$. Rosich is with the Interdisciplinary Centre for Security, Reliability and Trust (SnT) in the University of Luxembourg, Luxembourg albert.rosich at uni.lu

${ }^{2}$ V. Puig is with the Advanced Control Systems (SAC) Research Group, in the Universitat Politècnica de Catalunya, Catalonia, Spain vi cenc • puig at upc.edu in model form. The consistency is typically performed by means of residual computation.

The residual expressions are typically obtained from analytical redundancy relations derived from the combination of the system model equations and available known system variables (e.g. on-line control signals and/or sensor measurements). At each time instant, residuals are compared with a threshold value (zero in ideal case or almost zero in real case). The threshold value can be determined using statistical or set-based methods that take into account the effect of noise and model uncertainty [1]. Finally, faults can be diagnosed by checking inconsistencies through the threshold residuals and comparing the obtained residual signature with the fault signature matrix FSM [7].

The objective in this paper is to develop and apply an efficient method to detect and locate leaks in a water distribution network. The method is based on the analysis of the water network model with leaks in order to derive a special class of residuals generators with suitable properties for leak localization. Because the water network model is described by a set of non-linear equations with non-explicit solution, analytical residuals cannot be easily derived neither implemented. Thus, the proposed method differs from others in the fault model-based diagnosis literature in the sense that residuals are not analytically obtained, instead an algorithm that numerically computes the residuals is presented. Furthermore, the class of residuals designed in this paper presents leak decoupling properties which facilitate the localization of leaks. Finally, in order to highlight the applicability of the proposed leakage localization methodology, it will be tested in a benchmark water network where some sensors will be assumed already installed in the DMA and numerical results will be derived.

The organization of the paper is as follows: Section II presents the water network model when no leak is present as well as when leaks are taken into account. In this section, a numerical method to compute the water model is briefly reviewed. Section III introduces the principles of the proposed leak localization methodology and details the algorithm to compute the designed residuals. Section IV presents a diagnosis analysis in order to known beforehand which are the leaks that can be detected and located. The case study and results obtained through the proposed approach are shown in Section V. Finally, Section VI concludes the paper and suggests some future research lines to extend the proposed methodology. 


\section{WATER Distribution Network Model}

A water distribution network model is typically defined by a set of $N$ nodes representing the junctions or reservoirs, and a set of edges $E$ representing the pipes. Let $q_{e}$ for each $e \in E$ with $e=(i, j)$ be the flows from node $i$ to node $j$, and let $h_{n}$ for each $n \in N$ be the pressures. The set of pressures is partitioned into two disjoint subsets: the subset of junction pressures $U \subset N$ and the subset of reservoir pressures $K \subset$ $N$ (pressures at reservoirs are fixed and known by definition).

Water distribution networks are usually modelled assuming static models as follows [2]:

- Flow balance conservation in nodes,

$$
\sum_{n, j} q_{n j}=d_{n} \quad \text { for all } n \in U
$$

where $d_{n}$ is the demand at node $n$.

- Hasen-Williams pressure loss function in pipes,

$$
h_{i}-h_{j}=r_{e} q_{e}\left|q_{e}\right|^{\gamma_{e}} \quad \text { for all } e \in E ; e=(i, j)
$$

where $r_{e}$ is a pipe parameter which depends on diameter, roughness and length, and $\gamma_{e}$ is the flow exponent parameter.

- Reservoir pressure condition,

$$
h_{n}=h_{n}^{*} \quad \text { for all } n \in K
$$

where $h_{n}^{*}$ is a known pressure value.

Let $p_{u}, p_{k}$ and $p_{e}$ be the number of junction nodes, reservoirs and flows, respectively (i.e. $p_{u}=|U|, p_{k}=$ $|K|$ and $\left.p_{e}=|E|\right)$. and let $\mathbf{h}=\left(h_{1}, \ldots, h_{p_{u}}\right)^{T}, \mathbf{h}_{\mathbf{0}}=$ $\left(h_{1}^{*}, \ldots, h_{p_{k}}^{*}\right)^{T}, \mathbf{q}=\left(q_{1}, \ldots, q_{p_{e}}\right)^{T}$ and $\mathbf{d}=\left(d_{1}, \ldots, d_{p_{u}}\right)^{T}$ denote the vector of junction node pressures, reservoir pressures, flows and known demands, respectively. Then, the water network model can be formulated in matrix form as follows:

$$
\left(\begin{array}{cc}
A_{11}(\mathbf{q}) & A_{12} \\
A_{21} & 0
\end{array}\right)\left(\begin{array}{c}
\mathbf{q} \\
\mathbf{h}
\end{array}\right)=\left(\begin{array}{c}
-A_{10} \mathbf{h}_{\mathbf{0}} \\
\mathbf{d}
\end{array}\right)
$$

where $A_{11}(\mathbf{q})=\operatorname{diag}\left(r_{1}\left|q_{1}\right|^{\gamma_{1}}, \ldots, r_{p_{e}}\left|q_{p_{e}}\right|^{\gamma_{p_{e}}}\right), A_{12}=$ $A_{21}^{T}$, and $A_{10}=A_{01}^{T}$. The matrices $A_{21}$ and $A_{01}$ are the incidence matrices obtained from the network graph when only junction and reservoir nodes are regarded, respectively. The resulting water network model has $\mathbf{q}$ and $\mathbf{h}$ as unknowns, $p_{e}$ non-linear equations (pressure losses) and $p_{u}$ linear equations (flow balances).

\section{A. Adding leaks to the model}

A water leak could theoretically appear at any point of any pipe of the network which would imply that the graph structure of the model should be modified by adding a new junction node at the leaking point, furthermore new parameters should be derived for the leaking pipe. For this reason, the modeling of any possible leak becomes difficult and unfeasible in practice. To mitigate this problem, it is usually assumed that leaks can only appear at existing nodes [14]. Therefore, the set of leaks to be considered can be constrained by the number of existing junction nodes in the network. In addition, no new parameter estimation needs to be done since no change in the pipe is assumed. In this section, the model in (4) is extended by taking into account the possible leaks.

Let $\mathbf{l}=\left(l_{1}, \ldots, l_{p_{u}}\right)^{T}$ be the vector of possible leaks in the water network, then the model (4) can be extended to also include leak in nodes as

$$
\left(\begin{array}{ccc}
A_{11}(\mathbf{q}) & A_{12} & 0 \\
A_{21} & 0 & -I
\end{array}\right)\left(\begin{array}{c}
\mathbf{q} \\
\mathbf{h} \\
\mathbf{l}
\end{array}\right)=\left(\begin{array}{c}
-A_{10} \mathbf{h}_{\mathbf{0}} \\
\mathbf{d}
\end{array}\right)
$$

Usually, this model is implicitly used to study leak sensitivity analysis by means of simulations. For instance, assume that the effect of leak $i$ is studied for a specific known value $l_{i}$. Then, according to (5), this can be done by solving model (4) with $\mathbf{d}=\left(d_{1}, \ldots, d_{i}+l_{i}, \ldots, d_{p_{u}}\right)^{T}$.

\section{B. Model Solution}

The model in (4) is not linear and cannot be solved analytically. Therefore numerical tools are needed in order to provide a solution in terms of flows, $\mathbf{q}$, and pressures $\mathbf{h}$. In [17], it is proved that the solution for the model (4) exists and is unique. The proof is based on considering the following non-linear optimization problem,

$$
\min \left\{\sum_{i=1}^{n_{e}}\left(\int_{0}^{q_{i}} r_{i}|t|^{\gamma_{i}} d t\right)+\sum_{j=1}^{n_{k}} h_{j}^{*} \sum_{i=1}^{n_{e}} A_{10_{(i, j)}} q_{i}\right\}
$$

subject to:

$$
\sum_{i=1}^{n_{e}} A_{21_{(j, i)}} q_{i}-d_{j}=0 \quad \text { for } j=\left\{1, \ldots, n_{u}\right\}
$$

that is known as Content Model [4]. It can be shown that the solution of this optimization problem is given by (4). Since all $r_{i}$ and $\gamma_{i}$ are positives, the minimization problem is convex which means that the solution of both, the Content Model and the water network model (4), exists and is unique.

Besides, [17] presents a numerical method based on the recursive Newton-Raphson algorithm to solve the water network model (4). This algorithm can be summarized with the following two recursive equations:

$$
\begin{aligned}
\mathbf{h}_{(k+1)}= & -\left(A_{21} N^{-1} A_{11}\left(\mathbf{q}_{(k)}\right)^{-1} A_{12}\right)^{-1}\left(A _ { 2 1 } N ^ { - 1 } \left(\mathbf{q}_{(k)}+\right.\right. \\
& \left.\left.+A_{11}\left(\mathbf{q}_{(k)}\right)^{-1} A_{10} \mathbf{h}_{\mathbf{0}}\right)+\left(\mathbf{d}-A_{21} \mathbf{q}_{(k)}\right)\right) \\
\mathbf{q}_{(k+1)}= & \left(I-N^{-1}\right) \mathbf{q}_{(k)}-N^{-1} A_{11}\left(\mathbf{q}_{(k)}\right)\left(A_{12} \mathbf{h}_{(k)}\right)+ \\
& \left.+A_{10} \mathbf{h}_{\mathbf{0}}\right)
\end{aligned}
$$

where $N=\operatorname{diag}\left(\gamma_{1}, \gamma_{2}, \ldots, \gamma_{p_{e}}\right)$ and $A_{11}\left(\mathbf{q}_{(k)}\right)$ is computed using $\mathbf{q}_{(k)}$. Here, it will be assumed that no flow $q \in \mathbf{q}_{(k)}$ is zero (null flows would lead to an ill conditioned problem where network reconfiguration techniques should be used). The algorithm is initialized with any guess values, $\mathbf{h}_{(0)}$ and $\mathbf{q}_{(0)}$, and the solution is found by recursively applying equations (8)-(9) until a preestablished degree of tolerance is achieved.

This method is used in the EPANET simulator [16] where large water networks can be efficiently solved. 


\section{RESIDUAL IMPLEMENTATION}

The residual generator proposed in this paper basically consists in the comparison of a measured pressure, $m$, with its model-based estimation, $\tilde{h}$, i.e.

$$
r=m-\tilde{h}
$$

In [13], this residual generator is used by evaluating $\tilde{h}$ through EPANET because the non-liniarities of the model make impossible to derive any analytical equation from the model. For this reason, in the previous model-based approaches for leak localization (see for example, [14], [13]), all measured pressures are estimaed using the free-leak model (4) and then they are inserted in the residual generator (10). It is worth to point out that no measured pressure is used to simulate the model (only reservoir pressures, $h^{*}$, are used) whereas measured pressures are used for generating the residual (10), thus the number of residual is the same as the number of sensors. Because of meshed topology of distributed network, a leak in a given node usually affects all measurements (i.e. all residuals are sensitive to any leak in some degree). Thus, leak isolation should be performed by means of sensitivity analysis where the sensitivity of every residual, with respect to each possible leak, is determined by simulation. However, the residual sensitivity is not a constant value but depends on the leak magnitude, which worsen the diagnosability performance in case that different leak magnitudes are present in the system.

To mitigate this problem, an alternative approach is presented in the next section where each residual becomes decoupled from certain desired leak and thus the localization task is improved since it does not depend on the leak magnitude.

\section{A. Leak decoupling}

As it was shown in Section II-B, the model (4) can be solved and the solution is unique. Now, consider that a junction node pressure $h_{i} \in \mathbf{h}$ is measured. This means that the pressure $h_{i}$ becomes known and can be added to the vector of known pressures, i.e., $\tilde{\mathbf{h}}_{\mathbf{0}}[i]=\left(h_{1}^{*}, \ldots, h_{p_{k}}^{*}, h_{i}\right)^{T}$, and removed from the vector of unknown pressures, i.e., $\tilde{\mathbf{h}}[i]=\left(h_{1}, \ldots, h_{i-1}, h_{i+1}, \ldots, h_{p_{u}}\right)^{T}$. By doing this, the solution space is reduced in one dimension which means that now one equation is redundant and therefore not needed to compute the solution.

Proposition 1: Given the water network model in (4) with no null flows and assuming that one junction node pressure in known, then the model can be solved by (8)-(9) if any row $j$ from matrix $A_{21}$ and its corresponding demand $d_{j}$ are removed.

Proof: First, note that in order to keep the notation consistent in (8)-(9) when the pressure of the $i$-th node is known, the $i$-th columns of matrix $A_{12}$ must be removed and inserted into $A_{10}$. This only affects to the term $\left(A_{21} N^{-1} A_{11}^{-1} A_{12}\right)$ in (8) which needs to be invertible.

Matrices $N$ and $A_{11}$ are invertible (under non-null flow assumption) and do not depend on which pressures are known. Then, from graph theory, it is easy to show that matrix $A_{21}$ is an $m \times n$ dimension matrix with $m \leq n$ and $\operatorname{rank}\left(A_{21}\right)=m$. Consequently, matrix $A_{12}$ is an $n \times m$ matrix with full column rank. This means that no rank deficient matrix is obtained by removing rows in $A_{21}$ or columns in $A_{12}$. Therefore, the term will be invertible as long as one row is removed from $A_{21}$.

The demand $d_{j}$ must be also removed for coherence with the resulting model.

The resulting solvable model when $h_{i}$ is measured is represented by

$$
\left(\begin{array}{cc}
A_{11}(\mathbf{q}) & \tilde{A}_{12}[i] \\
\tilde{A}_{21}[j] & 0
\end{array}\right)\left(\begin{array}{c}
\mathbf{q} \\
\tilde{\mathbf{h}}[i]
\end{array}\right)=\left(\begin{array}{c}
-\tilde{A}_{10}[i] \tilde{\mathbf{h}}_{\mathbf{0}}[i] \\
\tilde{\mathbf{d}}[j]
\end{array}\right)
$$

where $\tilde{A}_{12}[i]$ and $\tilde{A}_{21}[j]$ are the matrix obtained by removing the $i$-th column in $A_{12}$ and the $j$-th row in $A_{21}$, respectively. $\tilde{A}_{10}$ corresponds to the concatenation of matrix $A_{10}$ with the $i$-th column of $A_{12}$, and $\tilde{\mathbf{d}}[j]=$ $\left(d_{1}, \ldots, d_{j-1}, d_{j+1}, \ldots, d_{p_{k}}\right)^{T}$.

It is important to note that the model obtained in (11) has no physical sense and thus cannot be implemented in a water network simulator as EPANET. However, this new model presents an advantageous property compared with the former model.

Proposition 2: Given $\tilde{\mathbf{h}}[i]$ computed with model (11), then any residual $r=h_{m}-\tilde{h}$ for $\tilde{h} \in \tilde{\mathbf{h}}[i]$ is decoupled from leak $l_{j}$.

Proof: The model (11) is extended to also include the leak $l_{j}$, i.e.

$$
\left(\begin{array}{ccc}
A_{11}(\mathbf{q}) & \tilde{A}_{12}[i] & 0 \\
\tilde{A}_{21}[j] & 0 & 0
\end{array}\right)\left(\begin{array}{c}
\mathbf{q} \\
\tilde{\mathbf{h}}[i] \\
l_{j}
\end{array}\right)=\left(\begin{array}{c}
-\tilde{A}_{10}[i] \tilde{\mathbf{h}}_{\mathbf{0}}[i] \\
\tilde{\mathbf{d}}[j]
\end{array}\right)
$$

where the effect of leak $l_{j}$ is null because flow balance equation concerning node $j$ has been removed according to the Proposition 1. Then, the proof consists in showing that the solution of the water model when there is a leak $l_{j}$ is also the solution of the model in (12).

Assume that $\overline{\mathbf{h}}=\left(\bar{h}_{1}, \ldots, \bar{h}_{p_{u}}\right)^{T}$ and $\overline{\mathbf{q}}=\left(\bar{q}_{1}, \ldots, \bar{q}_{p_{e}}\right)^{T}$ are the solutions of model (5) for any $\mathbf{l}$ such that $\mathbf{l}=$ $\left(0, \ldots, 0, l_{j}, 0, \ldots, 0\right)^{T}$. Then, from (12), we have that

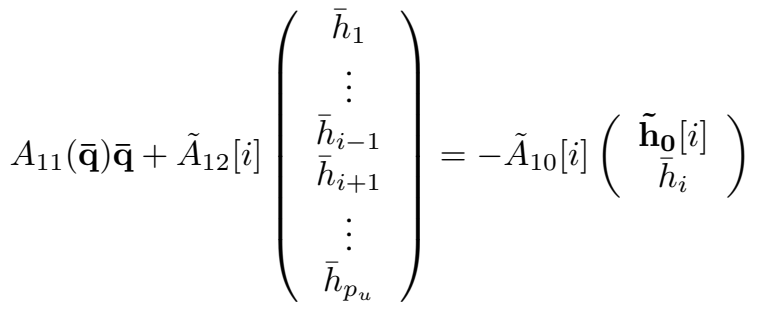

must hold since the equations are the same as in (5), and

$$
\tilde{A}_{21}[j] \overline{\mathbf{q}}=\tilde{\mathbf{d}}[j]
$$

must hold since it does not depend on $l_{j}$.

From this result, we can not guarantee that the residual sensitivity will be large enough. However, we will be able to implement a class of residuals which are insensitive to certain leak, independently of its magnitude. In case of measuring 
more than one junction node pressure, the same result could still be applied by removing more than one column and row. Then, the residual would be decoupled from more than one leak. In this approach, sensors can be classified in two sets according its purpose: sensors for leak decoupling, and sensors for residual generation.

\section{B. Automatic residual computation}

Another advantage of the proposed method for residual generation is that we can generate many residuals with few sensors. To illustrate this, consider now that there are two pressure sensors installed in the network. One sensor, placed in node $i$, is used to decouple the leak from the residual according to (11), whereas the other sensor, placed in node $k$, is used to generate the residual according to (10) (for indexes notational consistency, we need to assume without loss of generality that $i>k$ ). Since the $j$-th row in (11) has been arbitrary chosen, we can use the same sensor to decouple all the leaks $l_{j}$ for $j=\left\{1, \ldots, p_{u}\right\}$, obtaining $p_{u}$ different pressure estimates, $\left\{\tilde{\mathbf{h}}[i]_{1}, \ldots, \tilde{\mathbf{h}}[i]_{p_{u}}\right\}$. Then, $p_{u}$ residuals, each one insensitive to a different leak, can be computed by inserting the corresponding estimate in (10), i.e.,

$$
\begin{aligned}
& r_{j}=m_{k}-\tilde{h}_{k} \quad \text { for } \tilde{h}_{k} \in \tilde{\mathbf{h}}[i]_{j} ; \\
& j=\left\{1, \ldots, p_{u}\right\}
\end{aligned}
$$

Next algorithm summarizes the residual evaluation method presented in this paper.

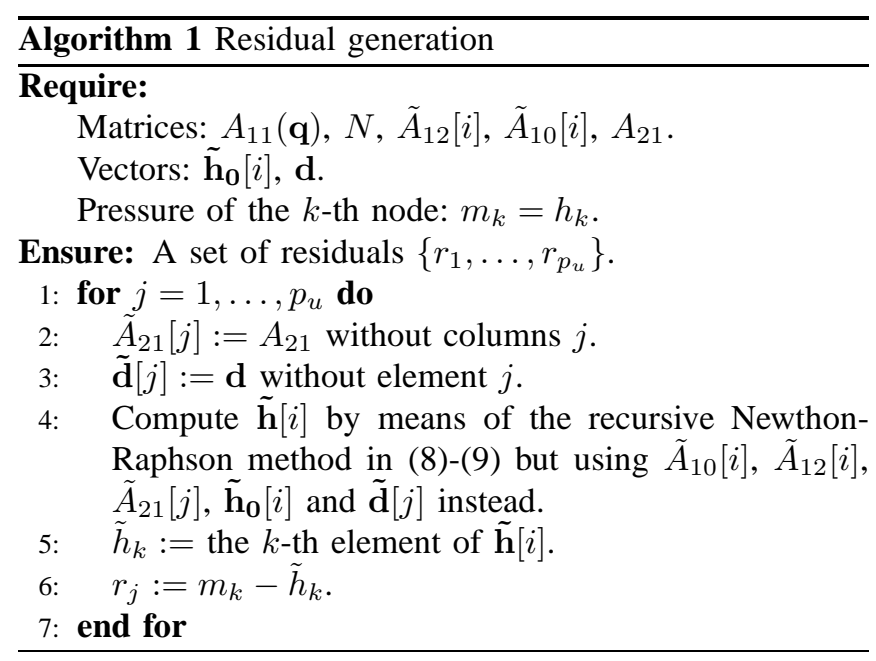

This algorithm could be executed on-line and be used as the residual generator core of the diagnosis system. Besides, since a generic water network model has been considered, the algorithm could be applied to any water distribution network system.

\section{LEAK LOCALIZATION ANALYSIS}

It is important to note that the insensitivity to leak $l_{j}$ of residual $r_{j}$ does not mean that the residual must be sensitive to the other leaks. Therefore, a diagnosis analysis is needed in order to determine the diagnosis performances expected from the residuals designed in Section III.
Because no analytical expression of the residual generator is available, the diagnosis analysis will be based on model properties. Here, with an abuse of notation, the vector of measured pressures is denoted by $\mathbf{m}=\left\{m_{1}, \ldots, m_{p_{m}}\right\} \subseteq$ $\mathbf{h}$, while the vector of unknown pressures is denoted by $\mathbf{u}=\left\{u_{1}, \ldots, u_{p_{u}-p_{m}}\right\}=\mathbf{h} \backslash \mathbf{m}$. Thus, the on-line information (observations) of the water network available for leak localization consists of the reservoir pressures vector, $\mathbf{h}_{\mathbf{0}}$, the demands vector $\mathbf{d}$ and the measured pressures vector, $\mathbf{m}$. Then, the set of observations consistent with the free-leak model is defined as

$$
\mathcal{O}_{\emptyset}=\left\{\left(\mathbf{h}_{\mathbf{0}}, \mathbf{d}, \mathbf{m}\right) \mid \exists \mathbf{u}, \mathbf{q}: \text { model (4) holds }\right\}
$$

Similarly, the set of observations consistent with a single leak, $l_{i}$, model can be defined as

$$
\begin{gathered}
\mathcal{O}_{l_{i}}=\left\{\left(\mathbf{h}_{\mathbf{0}}, \mathbf{d}, \mathbf{m}\right) \mid \exists \mathbf{u}, \mathbf{q}, \mathbf{l}: \text { model }(5)\right. \text { holds with } \\
\left.\mathbf{l}=\left(0, \ldots, 0, l_{i}, 0, \ldots, 0\right)^{T}\right\}
\end{gathered}
$$

Note that this definition can be easy extended to multiple leaks by allowing in (17) that multiple instance of vector 1 can take free values.

From the consistency observation sets, leak detectability is next defined.

Definition 1 (Leak detection): A leak $l_{j}$ is detectable if $\mathcal{O}_{l_{j}} \nsubseteq \mathcal{O}_{\emptyset}$

Equivalent definitions have been presented for fault detection (see e.g. [1] and [6]). In [12], fault detectability is presented in form of rank condition for linear dynamic systems. Here, we will derive analogous condition but using instead structural matrix properties since the model is nonlinear ${ }^{1}$. First, we rewrite the model in (5) as

$$
G \mathbf{x}+F \mathbf{l}=H \mathbf{y}
$$

$$
\begin{aligned}
& \text { where } \mathbf{x}=\left(\begin{array}{ll}
\mathbf{q}^{T} & \mathbf{u}^{T}
\end{array}\right)^{T}, \mathbf{y}=\left(\begin{array}{lll}
\mathbf{h}_{\mathbf{0}}^{T} & \mathbf{m}^{T} & \mathbf{d}^{T}
\end{array}\right)^{T} \text { and } \\
& G=\left(\begin{array}{cc}
A_{11}(\mathbf{q}) & A_{12}^{\prime} \\
A_{21} & 0
\end{array}\right), \quad F=\left(\begin{array}{c}
0 \\
-I
\end{array}\right), \\
& H=\left(\begin{array}{ccc}
-A_{10} & A_{12}^{\prime \prime} & 0 \\
0 & 0 & I
\end{array}\right)
\end{aligned}
$$

Matrices $A_{12}^{\prime}$ and $A_{12}^{\prime \prime}$ are constructed from $A_{12}$, by taking account the columns corresponding to $\mathbf{u}$ and $\mathbf{m}$, respectively.

Now, matrix $G$ is regarded as a structural matrix where all the entries of the diagonal matrix $A_{11}(\mathbf{q})$ are assumed algebraic independent coefficient [10]. Following the idea of [12] but applying it to the structural case, and taking into account Definition 1 , a leak $l_{i}$ is detectable if the following condition holds

$$
\operatorname{s-rank}\left(\left[G F_{j}\right]\right)>\operatorname{s-rank}(G)
$$

where s-rank denotes the structural rank and $F_{j}$ is the $j$ th column of matrix $F$. Note that, according to [10], the

\footnotetext{
${ }^{1}$ Another possibility, instead of using structural analysis, would be to consider the model linearized at some operating point, then the same results derived here from structural rank conditions will also be valid for standard rank as long as no flow is zero.
} 
structural rank is equivalent to the standard rank under the algebraic independent coefficient assumption.

Leak isolability is analogously defined from the set of consistency observations.

Definition 2 (Leak isolation): A leak $l_{j}$ is isolable from a leak $l_{k}$ if $\mathcal{O}_{l j} \nsubseteq \mathcal{O}_{l_{k}}$.

The definition of leak localization comes naturally from Definition 2.

Definition 3 (Leak localization): A leak $l_{j}$ can be located if it can be isolated from any other leak $l_{k}, j \neq k$.

According to [6], condition (19) can be extended to the isolability case. Therefore, a leak $l_{j}$ is isolable from a leak $l_{k}$ if

$$
\text { s-rank }\left(\left[\begin{array}{lll}
G & F_{j} & F_{k}
\end{array}\right]\right)>\mathrm{s}-\operatorname{rank}\left(\left[\begin{array}{ll}
G & F_{j}
\end{array}\right]\right)
$$

Diagnosis analysis can be performed by checking whether conditions (19) and (20) are fulfilled. Note that this analysis does not depend on the residual generator since it is based on model properties. However, it is easy to see that the model (11) used to derive a residual is consistent with this analysis presented in this section.

Proposition 3: The model in (11) can not be used to detect the leak $l_{j}$.

Proof: The corresponding $G$ matrix of model (11) is

$$
\left(\begin{array}{cc}
A_{11}(\mathbf{q}) & \tilde{A}_{12}[i] \\
\tilde{A}_{21}[j] & 0
\end{array}\right)
$$

whereas the corresponding $\left[G F_{j}\right]$ matrix of model (11) with leak $l_{j}$ is

$$
\left(\begin{array}{ccc}
A_{11}(\mathbf{q}) & \tilde{A}_{12}[i] & 0 \\
\tilde{A}_{21}[j] & 0 & 0
\end{array}\right)
$$

From this, it is easy to see that s-rank $\left(\left[\begin{array}{ll}G & F_{i}\end{array}\right]\right)=\operatorname{s-rank}(G)$ which means that leak $l_{j}$ cannot be detected.

This result reconfirm the fact that the decoupling property developed in Section III is given by the correct choice of the leak model for each residual computation.

\section{CASE Study: Hanoi WATER NETWORK}

The diagnosis analysis presented in Section IV and the residual developed in Section III will be implemented on the Hanoi water network benchmark [16] in order to illustrate the applicability of the proposed approach. Furthermore, the numerical results presented in this section will give us some insights on future possible extensions.

The Hanoi water network (see Figure 1) consists of 31 junction nodes, 1 reservoir node and 34 pipes. Here, we will assume that nodes 12 and 21 are measured and 31 leaks are considered (one leak for each junction node). How the result is affected by other possible sensor positions will be discussed later. Furthermore, the reservoir pressure and the demand in every node are known.

First, diagnosis analysis is performed. Condition (19) is satisfied for every single leak, therefore all leaks are detectable. Then, condition 2 is satisfied for any pair of leaks but $\left(l_{1}, l_{2}\right)$. Thus, every leak can be located except $l_{1}$ and $l_{2}$.

Equivalent results are obtained by analyzing detectability on the models used for residual generation. Table I shows, by

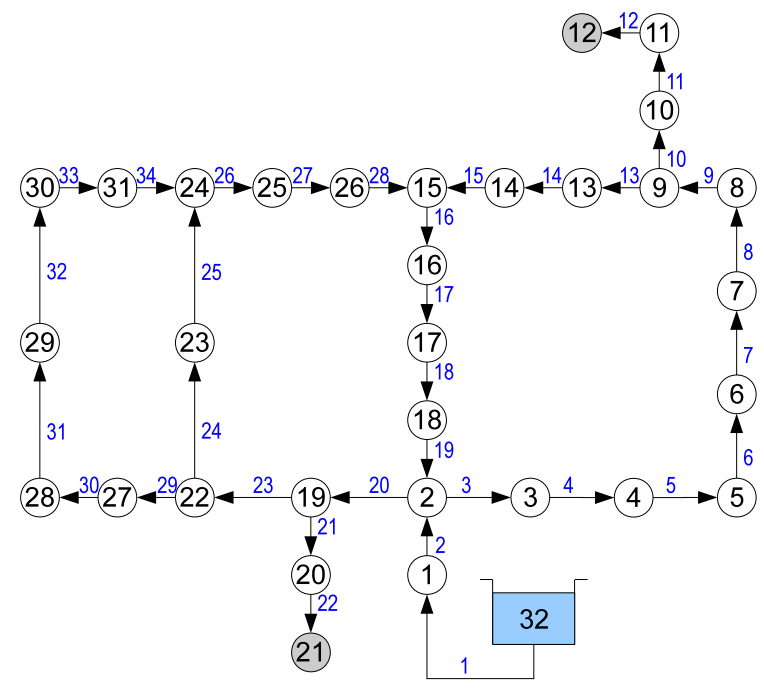

Fig. 1. Hanoi water network

TABLE I

THEORETICAL LEAK SIGNATURES

\begin{tabular}{c|ccccccc} 
& $l_{1}$ & $l_{2}$ & $l_{3}$ & $l_{4}$ & $\ldots$ & $l_{30}$ & $l_{31}$ \\
\hline$r_{1}$ & 0 & 0 & $\times$ & $\times$ & $\ldots$ & $\times$ & $\times$ \\
$r_{2}$ & 0 & 0 & $\times$ & $\times$ & $\ldots$ & $\times$ & $\times$ \\
$r_{3}$ & $\times$ & $\times$ & 0 & $\times$ & $\ldots$ & $\times$ & $\times$ \\
$r_{4}$ & $\times$ & $\times$ & $\times$ & 0 & & $\times$ & $\times$ \\
$\vdots$ & & $\vdots$ & & & $\ddots$ & & $\vdots$ \\
$r_{30}$ & $\times$ & $\times$ & $\times$ & $\times$ & & 0 & $\times$ \\
$r_{31}$ & $\times$ & $\times$ & $\times$ & $\times$ & $\ldots$ & $\times$ & 0
\end{tabular}

means of a cross symbol, which leaks can be detected from each residual. Note that, according to this table, all leaks can be detected and further all pair of leak can be isolated, except the pair $\left(l_{1}, l_{2}\right)$ which share the same leak signature.

At this point, it is worth to mention that other sensor positions would lead to other diagnosis capabilities. For instance, the leaks $\left\{l_{9}, l_{10}, l_{11}, l_{12}\right\}$ and $\left\{l_{19}, l_{20}, l_{21}\right\}$ would not be respectively isolable among them if the two sensors were placed in loop graph nodes, i.e., nodes $\{2, \ldots, 19,22, \ldots, 31\}$. This have a physical interpretation which is that sensors placed in upstream nodes "see" the same information for different downstream node leaks. Thus, it is convenient to place sensors in the extremes of the graph where water cannot flow to other downstream nodes.

Now, the residuals are implemented and computed under four leak scenarios in order to study their behavior. The considered scenarios are: i) no leak, ii) leak $l_{1}$, iii) leak $l_{10}$ and iv) leak $l_{22}$. The leak magnitude is set to the $10 \%$ of the demand in the corresponding leaking node, i.e., $l_{j}=0.1 d_{j}$. The results of the 31 residual responses for each studied scenario are depicted in Table II. Because the residuals are computed numerically (Newton-Raphson algorithm stops iterating when a preestablished tolerance is achieved), no exact zero is expected. However, the residuals that are not affected by the leak remains several orders of magnitude lower than the affected residuals, which make very easy to 
TABLE II

RESIDUAL RESPONSES

\begin{tabular}{|c|c|c|c|c|}
\hline & no leak & leak $l_{1}$ & leak $l_{10}$ & leak $l_{22}$ \\
\hline$r_{1}$ & $-2 \times 10^{-13}$ & $9 \times 10^{-13}$ & $4 \times 10^{-1}$ & $-2 \times 10^{-1}$ \\
\hline$r_{2}$ & $8 \times 10^{-9}$ & $8 \times 10^{-9}$ & $4 \times 10^{-1}$ & $-2 \times 10^{-1}$ \\
\hline$r_{3}$ & $-9 \times 10^{-7}$ & $-7 \times 10^{-3}$ & $3 \times 10^{-1}$ & $-4 \times 10^{-1}$ \\
\hline$r_{4}$ & $-6 \times 10^{-7}$ & $-1 \times 10^{-2}$ & $2 \times 10^{-1}$ & $-7 \times 10^{-1}$ \\
\hline$r_{5}$ & $-6 \times 10^{-7}$ & $-2 \times 10^{-2}$ & $2 \times 10^{-1}$ & $-1 \times 10^{0}$ \\
\hline$r_{6}$ & $-7 \times 10^{-7}$ & $-2 \times 10^{-2}$ & $1 \times 10^{-1}$ & $-1 \times 10^{0}$ \\
\hline$r_{7}$ & $-9 \times 10^{-7}$ & $-3 \times 10^{-2}$ & $1 \times 10^{-1}$ & $-1 \times 10^{0}$ \\
\hline$r_{8}$ & $-1 \times 10^{-6}$ & $-3 \times 10^{-2}$ & $1 \times 10^{-1}$ & $-1 \times 10^{0}$ \\
\hline$r_{9}$ & $-1 \times 10^{-6}$ & $-4 \times 10^{-2}$ & $8 \times 10^{-2}$ & $-1 \times 10^{0}$ \\
\hline$r_{10}$ & $-1 \times 10^{-6}$ & $-5 \times 10^{-2}$ & $5 \times 10^{-7}$ & $-1 \times 10^{0}$ \\
\hline$r_{11}$ & $-2 \times 10^{-6}$ & $-8 \times 10^{-2}$ & $-2 \times 10^{-1}$ & $-2 \times 10^{0}$ \\
\hline$r_{12}$ & $-4 \times 10^{-6}$ & $-1 \times 10^{-1}$ & $-7 \times 10^{-1}$ & $-4 \times 10^{0}$ \\
\hline & $-5 \times 10^{-7}$ & $-3 \times 10^{-2}$ & $1 \times 10^{-1}$ & $-1 \times 10^{0}$ \\
\hline 14 & $-1 \times 10^{-6}$ & $-1 \times 10^{-2}$ & $3 \times 10^{-1}$ & $-6 \times 10^{-1}$ \\
\hline$r_{15}$ & $-1 \times 10^{-7}$ & $-5 \times 10^{-3}$ & $3 \times 10^{-1}$ & $-4 \times 10^{-1}$ \\
\hline$r_{16}$ & $2 \times 10^{-7}$ & $-1 \times 10^{-3}$ & $4 \times 10^{-1}$ & $-3 \times 10^{-1}$ \\
\hline$r_{17}$ & $-1 \times 10^{-7}$ & $-9 \times 10^{-4}$ & $4 \times 10^{-1}$ & $-2 \times 10^{-1}$ \\
\hline$r_{18}$ & $-7 \times 10^{-7}$ & $-1 \times 10^{-4}$ & $4 \times 10^{-1}$ & $2 \times 10^{-1}$ \\
\hline$r_{19}$ & $1 \times 10^{-8}$ & $1 \times 10^{-2}$ & $5 \times 10^{-1}$ & $3 \times 10^{-2}$ \\
\hline$r_{20}$ & $-1 \times 10^{-7}$ & $2 \times 10^{-2}$ & $6 \times 10^{-1}$ & $4 \times 10^{-1}$ \\
\hline 21 & $-2 \times 10^{-7}$ & $2 \times 10^{-3}$ & $6 \times 10^{-1}$ & $-2 \times 10^{-1}$ \\
\hline$r_{22}$ & $-8 \times 10^{-8}$ & $1 \times 10^{-3}$ & $5 \times 10^{-1}$ & $-3 \times 10^{-8}$ \\
\hline$r_{2}$ & $-1 \times 10^{-7}$ & $8 \times 10^{-3}$ & $4 \times 10^{-1}$ & $-3 \times 10^{-2}$ \\
\hline$r_{24}$ & $-1 \times 10^{-7}$ & $7 \times 10^{-3}$ & $4 \times 10^{-1}$ & $-6 \times 10^{-2}$ \\
\hline$r_{25}$ & $-1 \times 10^{-7}$ & $5 \times 10^{-3}$ & $4 \times 10^{-1}$ & $-1 \times 10^{-1}$ \\
\hline$r_{26}$ & $3 \times 10^{-7}$ & $1 \times 10^{-3}$ & $4 \times 10^{-1}$ & $-2 \times 10^{-1}$ \\
\hline$r_{27}$ & $-1 \times 10^{-7}$ & $9 \times 10^{-3}$ & $5 \times 10^{-1}$ & $-1 \times 10^{-2}$ \\
\hline$r_{28}$ & $-5 \times 10^{-8}$ & $8 \times 10^{-3}$ & $4 \times 10^{-1}$ & $-5 \times 10^{-2}$ \\
\hline$r_{29}$ & $-1 \times 10^{-7}$ & $8 \times 10^{-3}$ & $4 \times 10^{-1}$ & $-5 \times 10^{-2}$ \\
\hline$r_{30}$ & $3 \times 10^{-7}$ & $7 \times 10^{-3}$ & $4 \times 10^{-1}$ & $-6 \times 10^{-2}$ \\
\hline$r_{31}$ & $2 \times 10^{-8}$ & $7 \times 10^{-3}$ & $4 \times 10^{-1}$ & $-6 \times 10^{-2}$ \\
\hline
\end{tabular}

determine whether a residual has exceed its threshold. Bold numbers in Table II indicate residuals not exceeding their threshold.

It is also important to note that, in the case of leak $l_{1}$ scenario, residual $r_{1}$ is not sensitive to $l_{1}$ by design, whereas residual $r_{2}$ becomes insensitive to $l_{1}$ as a consequence of the system structure, i.e. s-rank $\left(\left[\begin{array}{lll}G & F_{1} & F_{2}\end{array}\right]\right)=\operatorname{s-rank}\left(\left[\begin{array}{ll}G & F_{1}\end{array}\right]\right)$. This is something that could not avoided during the residual design in Section III. Nevertheless, the remaining leaks can perfectly be located according to the leak signatures in Table I where the diagonal of zeros, given by the residual design, plays a crucial role.

\section{CONCLUSION}

Up to now, because the non-linearities of the water network, only sensitivity analysis was been considered for leak diagnosis in previous works. The present paper goes one step further on the problem of leak localization. Here, a new class of structured residuals, computed numerically, allows to qualitative improve the isolability by decoupling the residuals from specific leaks. Another advantage, in favor of the proposed approach, is that the residuals can be computed efficiently. Here, for the sake of space limitation, a small example has been presented. However the numerical tool used to compute the residuals is nowadays widely used to solve water network models involving thousands of nodes. Anyway, in case that computational time was an issue, the approach could be extended in a decentralized fashion were not all residuals would be computed at once but some of them, and depending on their responses a new set would be selected for computation and so on until the correct leak was located.

The presented work assumes that demands are known and the measurement are exact which is not real in practice. Demands are usually estimated from population consumer patterns which are never exact and real pressure sensors are imprecise. This, together with some parameters inaccuracies, makes that uncertainties should be taken into account for a real implementation. Future research can go in this direction where interval methods or constraints satisfaction tools [8] could be considered.

As mentioned before, in this approach sensors can be used for two different purposes: leak decoupling and residual generation. This poses a trade-off in the sense that the larger number of sensor for leak decoupling is used, the less residuals are obtained. Moreover, the diagnosis performance depends on the positions and the number of sensors. Therefore, further work should be done in order to known which sensors should be installed and for which purpose.

\section{REFERENCES}

[1] M. Blanke, M. Kinnaert, J. Lunze, and M. Staroswiecki. Diagnosis and Fault-Tolerant Control. Springer, 2nd edition, 2006.

[2] Ulanicki Brdys. Operational control of water systems. Prentice Hall International, Prentice Hall International, 1994.

[3] P.B. Cheung, G. V. Girol, N. Abe, and M. Propato. Integrating water systems. chapter Night flow analysis and modeling for leakage estimation in a water distribution system, pages 509-513. Taylor and Francis Group, London, UK, 2009.

[4] M. Collins, L. Cooper, R. Helgason, J. Kennington, and L. LeBlanc. Solving the pipe network analysis problem using optimization techniques. Management Science, 24(7):747-760, 1978.

[5] A.F. Colombo, P. Lee, and B.W. Karney. A selective literature review of transient-based leak detection methods. Journal of Hydroenvironment Research, 2(2-4):212-227, April 2009.

[6] Erik Frisk, Mattias Krysander, and Jan Åslund. Sensor placement for fault isolation in linear differential-algebraic systems. Automatica, 45(2):364-371, 2009.

[7] Janos Gertler. Fault Detection and Diagnosis in Engineering Systems. Marcel Dekker, Inc., New York, 1998.

[8] Jaulin L., M. Kieffer, O. Didrit, and E. Walter. Applied Interval Analysis with Examples in Parameter and State Estimation, Robust Control and Robotics. Springer-Verlag, 2001.

[9] A. Lambert. Accounting for losses: The bursts and background concept. Water and Environment Journal, 8(2):205-214, April 1994.

[10] K. Murota. Matrices and Matroids for Systems Analysis. Springer, 2000.

[11] Bedjaoui N. and Weyer E. Algorithms for leak detection, estimation, isolation and localization in open water channels. Control Engineering Practice, 19(6):564-573, June 2011.

[12] M. Nyberg and E. Frisk. Residual generation for fault diagnosis of systems described by linear differential-algebraic equations. Automatic Control, IEEE Transactions on, 51(12):1995 -2000, dec. 2006.

[13] R. Pérez, V. Puig, J. Pascual, J. Quevedo, and E. Landeros. Methodology for leakage isolation using pressure sensitivity analysis in water distribution networks. Control Engineering Practice, 19(10):11571167, October 2011.

[14] Ligget Pudar. Leaks in pipe networks. Journal of Hydraulic Eng., 118(7):1031-1046, 1992.

[15] J. Ragot and D. Maquin. Fault measurement detection in an urban water supply network. Journal of Process Control, 16(9):887-902, April 2006.

[16] L.A. Rossman. Epanet 2 user's manual. United States Envionmental Protection Agency, 2000.

[17] E. Todini and S. Pilati. Computer applications in water supply: vol. 1systems analysis and simulation. chapter A gradient algorithm for the analysis of pipe networks, pages 1-20. Research Studies Press Ltd., Taunton, UK, UK, 1988. 